\title{
Triage and Management of Accidental Laboratory Exposures to Biosafety Level-3 AND -4 Agents
}

Peter Jahrling, Colleen Rodak, Mike Bray, and Richard T. Davey

The recent expansion of biocontainment laboratory capacity in the United States has drawn attention to the possibility of occupational exposures to BSL-3 and -4 agents and has prompted a reassessment of medical management procedures and facilities to deal with these contingencies. A workshop hosted by the National Interagency Biodefense Campus was held in October 2007 and was attended by representatives of all existing and planned BSL-4 research facilities in the U.S. and Canada. This report summarizes important points of discussion and recommendations for future coordinated action, including guidelines for the engineering and operational controls appropriate for a hospital care and isolation unit. Recommendations pertained to initial management of exposures (ie, immediate treatment of penetrating injuries, reporting of exposures, initial evaluation, and triage). Isolation and medical care in a referral hospital (including minimum standards for isolation units), staff recruitment and training, and community outreach also were addressed. Workshop participants agreed that any unit designated for the isolation and treatment of laboratory employees accidentally infected with a BSL-3 or -4 pathogen should be designed to maximize the efficacy of patient care while minimizing the risk of transmission of infection. Further, participants concurred that there is no medically based rationale for building care and isolation units to standards approximating a BSL-4 laboratory. Instead, laboratory workers accidentally exposed to pathogens should be cared for in hospital isolation suites staffed by highly trained professionals following strict infection control procedures.

$\mathrm{T}$ he CONSTRUCtion of a nUmber of new federally funded biocontainment laboratories in response to the 2001 terror attacks, in compliance with Homeland Security Presidential Directives 10 and $18,{ }^{1,2}$ has raised concerns that a significant expansion in the laboratory workforce will result in an increased number of accidental exposures, some of which might lead to actual infection. ${ }^{3}$ While it is true that accidental infections of laboratory workers studying pathogenic bacteria and viruses were at one time fairly common, their incidence has been markedly reduced as a result of the standardization of laboratory design, biosafety practices, and employee training, so that only a handful of cases have occurred in the past few decades. ${ }^{4}$
Much of this new capacity in Biosafety Level-4 (BSL-4) biocontainment laboratories will be centered on the National Interagency Biodefense Campus (NIBC) at Fort Detrick, which includes the existing United States Army Medical Research Institute of Infectious Diseases (USAMRIID) and a planned expansion, plus the National Institute of Allergy and Infectious Diseases (NIAID) Integrated Research Facility (NIAID-IRF) and a new Department of Homeland Security (DHS) laboratory, the National Biodefense Analysis and Countermeasures Center (NBACC). NIH also has upgraded the laboratory capacity at its Rocky Mountain Laboratories in Hamilton, Montana, by expanding the amount of Biosafety Level-3 (BSL-3)

Peter Jahrling, PhD, is Director, Office of the Chief Scientist, and Mike Bray, MD, MPH, is Medical Officer, both at the Integrated Research Facility, National Institute of Allergy and Infectious Diseases (NIAID), Frederick, Maryland. Colleen Rodak, MSN, is Associate Clinical Director, and Richard T. Davey, MD, is Deputy Director, both in the Division of Clinical Research, NIAID, Bethesda, MD. 


\section{Workshop Participants}

Department of Defense, Fort Detrick, MD

LTC Robert M. Gum

LTC Carl G. Hover

Mike Jewett

\section{USAMRIID, Fort Detrick, MD}

Ellen F. Boudreau, MD

Mark Kortepeter, MD, MPH

George V. Ludwig, PhD

Phillip R. Pittman, MD

Janice M. Rusnak, MD

COL John P. Skvorak

\section{CDC, Atlanta, GA}

L. Casey Chosewood, MD

Eileen Farnon, MD

Thomas Ksiazek, DVM, PhD

Eli W. Warnock III, MD

NIH, Bethesda, MD

Thomas C. Arminio

Michael Banyas

Karyl S. Barron, MD

John Beigel, MD

Marshall Bloom, MD

Nancy Boyd

Mike Bray, MD, MPH

Linda Coe, RN, MSN

Lisa L. Coronado

Richard Davey, Jr., MD

Michele R. Evans, PhD

Nancy P. Hoe, PhD

David K. Henderson, MD

Peter B. Jahrling, PhD

Scott D. Keimig

Jean P. Khoshbin, RA

H. Clifford Lane, MD

James V. Lawler, MD, MPH

Farhad Memarzadeh, PhD

Tara N. Palmore, MD

Alberta J. Peugeot, FNP

Colleen Rodak, MSN

Jorge A. Tavel, MD

Deborah E. Wilson, MD
Mary Wright, MD, MPH

Kathryn C. Zoon, PhD

Department of Homeland Security
Kevin Anderson, PhD
Bernard Courtney, PhD
James Estep, DVM, PhD
J. Patrick Fitch, PhD

Canadian National Centre for Foreign

Animal Disease

John Copps, PhD

Public Health Agency of Canada

Heinz Feldmann, MD

Eleanor Percy, RN

Boston University

Ellen Berlin

Larry Culpepper, MD, MPH

Mark S. Klempner, MD

Carol A. Sulis, MD

Ara Tahmassian

Emory University Hospital

Bruce Ribner, MD, MPH

Georgetown University

Daniel Lucey, MD, MPH

\section{Southwest Foundation for Biomedical}

Research

Jean L. Patterson, PhD

Saint Patrick's Hospital, Missoula, MT

George Risi, MD

U of Nebraska Medical Center

Philip Smith, MD

U of Texas Medical Branch Galveston

Nelson Avery, MD

Miguel Grimaldo

Michael R. Holbrook, PhD

Domenica M. Zimmerman space and adding a new BSL-4 lab, and it is supporting the construction of National Biocontainment Laboratories at Boston University and at the University of Texas Medical Branch, Galveston, both of which will contain BSL-3 and -4 units. In anticipation of public concerns, the NIBC Executive Steering Committee tasked its Scientific Interac- tions Subcommittee with organizing a workshop to review procedures for dealing with accidental exposures in laboratories currently conducting research on highly pathogenic (BSL-3 and -4) agents and to recommend optimal strategies for their detection and management in the future expanded biodefense research community. 
The workshop took place in Bethesda, Maryland, on October 25-26, 2007. It was attended by representatives of the National Institutes of Health (NIH), the Centers for Disease Control and Prevention (CDC) in Atlanta, USAMRIID and other Department of Defense organizations at Fort Detrick, the Department of Homeland Security and 2 Canadian government laboratories, and by physicians, nurses, biosafety officers, and other personnel from a number of U.S. universities and hospitals that currently perform or support BSL-3 and -4 research (see sidebar for participant list). This report summarizes important points of discussion and recommendations for future coordinated action, including guidelines for the engineering and operational controls appropriate for a care and isolation unit for the treatment of infected laboratory workers in North America. These guidelines join with recently published guidelines by the 16-member European Network of Infectious Diseases (EUNID) for the design and operation of high-level isolation units (HLIU) in those national or regional sites in Europe also actively engaged in research that involves a risk of exposure to potential bioterror agents or emerging pathogens. ${ }^{5}$

\section{BACKGROUND ON BSL-3 AND -4 RESEARCH}

Government-sponsored research on virulent microbes such as the agents of smallpox, tularemia, and plague has been conducted for more than a century, but efforts to develop standard approaches to pathogen classification and laboratory biosafety did not begin until the 1970s, with the publication by the CDC of Classification of Etiologic Agents on the Basis of Hazard. $^{6}$ That volume was followed in 1984 by the first laboratory biosafety manual, Biosafety in Microbiological and Biomedical Laboratories, which is now in its fifth edition. ${ }^{7}$

Under the CDC classification system, research on each species of infectious agent is assigned to 1 of 4 biosafety levels, based on the threat the research poses to laboratory workers, especially when exposure occurs by the aerosol route. Agents that are innocuous for humans may be studied using BSL-1 practices, which do not require special facility design or laboratory equipment to contain aerosols. Microbes such as measles or seasonal influenza viruses, which usually cause mild illness in humans that can be prevented by vaccination or treated with approved forms of therapy, are studied at BSL-2, which is characterized by more extensive personnel training, the use of biosafety cabinets to prevent aerosol exposure, and other measures. Research on more virulent agents, such as aerosolized Bacillus anthracis, which can cause lethal disease in humans but for which effective countermeasures exist in the form of vaccines and antibiotics, requires BSL-3 practices, which include restricted laboratory access, performance of all manipulations in biosafety cabinets, and the use of personal protective equipment. BSL- 4 is reserved for research on agents such as Ebola virus, which cause severe illness in humans that currently cannot be prevented by vaccination or that does not respond to treatment with any currently available medications. Although some laboratories employ hood lines ("glove boxes") for this purpose, the majority now use a "space suit" approach, in which all personnel wear positive-pressure plastic suits to prevent any possible aerosol exposure. Researchers follow BSL-3 practices in their work, including confining all infectious materials to biological safety cabinets or other primary containment devices, plus other special procedures unique to the BSL-4 environment.

Until recently, there were only a few BSL-4 laboratories, while studies on BSL-3 pathogens were carried out at a larger number of facilities. However, as a consequence of the terror attacks of September 11, 2001, the subsequent mailing of letters containing spores of Bacillus anthracis, and increasing awareness of the threat of emerging infectious diseases, the federal government has funded the construction of a number of new laboratories with combined BSL-3 and BSL-4 (BSL$(3+4))$ capabilities. This expansion of capacity will undoubtedly enhance the defense posture of the nation to deal with a biological attack or an emerging infectious disease, as detailed in HSPD-10 and -18. However, challenges remain in training the workforce ${ }^{8}$ and in dealing with occupational exposures should they occur.

\section{Organization of THE WORKSHOP}

The NIBC-sponsored workshop brought together leading personnel from laboratories that are currently performing BSL- $(3+4)$ research and laboratories that are now under construction and will begin operation in the near future. Before the workshop convened, representatives of the currently active labs completed a detailed questionnaire regarding their procedures for detecting and responding to accidental exposures. The questionnaire elicited information on the following topics:

- Preliminary medical screening of laboratory workers

- Evaluation of unexplained illnesses in lab personnel

- Initial management of accidental exposures, including

- Immediate treatment of penetrating injuries

- Reporting of exposures

- Initial evaluation

- Isolation and treatment, including

- Medical care in a referral hospital

- Minimal standards for care and isolation units

Staff recruitment and training

- Community outreach by the medical care facility

- Future initiatives

Responses to the questionnaires were tabulated, summarized, and presented to all participants at the beginning of the meeting, so that their experience could be used as the basis for a coordinated approach by all members of the 
future expanded biodefense research community. Participants then divided into working groups that focused on individual topics in the above outline; each group produced a series of recommendations that were then discussed and revised in a manner generally acceptable to the participants. The remainder of this report consists of concise summaries of questionnaire responses, working group discussions, and recommendations for future action.

\section{Preliminary Medical Screening AND VACCINATION}

All currently operational BSL-(3+4) laboratories screen their new employees by eliciting a comprehensive medical history and performing a medical examination to detect any underlying illness or a potential predisposition to acquiring infection, as suggested by the BMBL. ${ }^{7}$ Most labs also collect and retain a serum sample that can potentially be used to provide serologic evidence of the employee's response to vaccination or to serve as a baseline sample in the event of an accidental exposure to an infectious agent. Currently, active laboratories also vaccinate their new employees, when vaccines are available, against the pathogens that are the object of their research. The center of this activity to date has been the Special Immunization Program at USAMRIID, which has administered a number of vaccines under Investigational New Drug (IND) procedures to its own employees and those of other laboratories.

\section{Recommendation:}

1. Standards should be developed for pre-employment medical health examinations of prospective employees of BSL- $(3+4)$ laboratories, including checklists for behavioral health screening.

Behavioral health screening involves screening for alcohol and drug abuse, anxiety and depression, likelihood that the employee will follow safety rules, and the employee's general attitudes toward safety.

IND vaccines that could protect laboratory workers against a range of BSL-3 and -4 pathogens have been developed, but their high cost and limited availability are major issues for new BSL- $(3+4)$ laboratories; policymakers should address this issue as soon as possible.?

\section{Evaluation of UneXPLAined Illnesses}

An exposure to a pathogen in a BSL- $(3+4)$ laboratory might come to the attention of medical professionals in 1 of 3 ways: (1) an accident such as an animal bite or a skin puncture by a contaminated needle that the employee reports to a biosafety officer; (2) a similar event that the employee recognizes but fails to report; or (3) an unrecognized exposure, such as contact with infectious material that has been removed from biocontainment without adequate disinfection or labeling. In the latter 2 cases, the occurrence of the exposure may be recognized only when the employee becomes ill. It is therefore essential to monitor the health of all employees for the occurrence of illnesses that cannot be explained by chains of transmission, such as a seasonal influenza outbreak.

All currently operational BSL- $(3+4)$ laboratories represented at the workshop instruct their workers to monitor their own health status and require supervisors to track attendance and respond to any unexpected absences. Because the initial signs and symptoms of severe laboratory-acquired infections may resemble those of mundane illnesses such as colds, influenza, or gastroenteritis, any unexpected illness or absence from the workplace requires careful assessment.

\section{Recommendations:}

1. All laboratory personnel should be trained to recognize the signs and symptoms of illnesses typically caused by the agents with which they are working. Emergency 24-hour medical contact information should be readily available on wall postings and in a form that can be conveniently carried, such as a wallet card.

2. Employees should be required to phone in when absent from work for any illness and should be strongly encouraged to voluntarily report the nature of their illness. A mechanism for $24 / 7$ reporting should be in place. The overall system of reporting and response should be designed to ensure that no punitive action will be taken.

3. Standard procedures should be in place for supervisors to detect absences, for how long an absence may continue before reporting is required, and how and by whom an unexpected absence is to be investigated and managed once it is detected.

\section{Initial Management of Accidental EXPOSURES}

Lengthy experience has proven that the risk of exposure to infectious material in BSL- $(3+4)$ laboratories is markedly reduced through proper facility design and the use of personal protective equipment, biosafety cabinets, disinfectants, and other measures and rigorous adherence to good work practices. Nevertheless, it is impossible to rule out the rare occurrence of accidental exposures through needle punctures, animal bites, or airborne, skin, or mucous membrane contact with contaminated material. All currently operational BSL- $(3+4)$ laboratories were therefore polled to elicit their plans for managing accidental exposures, so that their experience could be used as a knowledge base for the future expanded biodefense community. 


\section{Immediate Treatment of Penetrating Injuries}

Although hypodermic needles, scalpels, and other "sharps" pose a hazard for laboratory workers, their use is unavoidable for a range of procedures, especially for studies in animals. Animal studies also involve a small but non-zero risk of bites or scratches. All currently operational BSL$(3+4)$ laboratories therefore instruct their employees in the steps to take in the event of a "sharps" injury or an animal bite, including immediate wound cleansing.

\section{Recommendations:}

1. First aid guidelines should be reviewed regularly and improved as appropriate, based on scientifically sound information and the results of simulation studies in laboratory animals. For example, although washing a wound with soap and water for 5 minutes has traditionally been recommended to reduce the risk of infection, the optimal period of washing has not been scientifically determined in the case of highly virulent pathogens.

2. Standard protocols or algorithms for the immediate response to accidental exposure to specific pathogens should include the development of agent-specific kits containing appropriate medications and directions for their use.

\section{Reporting an Exposure}

The standard operating procedures of all currently operational laboratories represented at the workshop enjoin employees to report immediately to their biosafety officer any known or possible exposure to an infectious agent. However, discussions brought out the fact that workers may be reluctant to report exposures, either because of fear of disciplinary action, the desire to keep a clean work record, uncertainty as to whether an exposure actually took place, or "wishful thinking" by the employee that his or her risk of acquiring infection after a particular exposure is minimal.

\section{Recommendations:}

1. To maximize compliance, procedures for reporting possible exposures should be clearly delineated and made as streamlined as possible, so that they are not viewed as too time-consuming or onerous. There must be a mechanism for 24/7 reporting.

2. The establishment of a nonpunitive, supportive atmosphere is key to ensuring that workers are willing to report exposures. Reporting should be recognized as a team effort that enhances the safety of the lab for everyone. Initial and refresher training of lab personnel is critical for success.

\section{Initial Evaluation and Triage}

All currently operational BSL- $(3+4)$ laboratories have developed general procedures for detecting and responding to accidental exposures to the pathogens they are studying. Discussion at the workshop revealed that a number of laboratories are also developing pathogen-specific algorithms for postexposure management.

\section{Recommendations:}

1. All BSL- $(3+4)$ laboratories would benefit from wellconsidered, comprehensive, and standardized procedures for the initial evaluation of occupational exposures. The process should be conducted by a qualified multidisciplinary group that includes the laboratory supervisor and biosafety officer, occupational health personnel, and a physician. When the patient is to be hospitalized, the group should also include representatives of the team that will be responsible for the subsequent care of the patient. Ideally, to avoid any conflict of interest, the team should include at least 1 medical professional with knowledge of the field but no vested interest in the work of the lab.

2. In evaluating a potential occupational exposure, the team should consider the specific pathogen(s) involved, the route and magnitude of exposure, the rapidity and thoroughness of local decontamination, the rapidity with which first aid was administered, the incubation period and transmissibility potential of the pathogen, and, where indicated, the value of prophylactic medications.

3. Administration of care should begin as soon as possible. Depending on circumstances, the laboratory worker exposed to a pathogen may require prompt isolation or may be discharged to home with expectant observation and early reassessment. The team also will be responsible for assessing situations in which a worker in biocontainment becomes ill from an unrelated medical condition (such as a heart attack or stroke) that requires immediate hospitalization without isolation.

4. Procedures to extricate and evacuate an injured worker from biocontainment should be in place for all labs. A working relationship with local first responders, who may be reluctant to enter the BSL- $(3+4)$ facility, should be initiated and maintained through regular training sessions. These procedures should be practiced on a periodic basis.

5. The legal and logistical issues associated with transporting an exposed individual from the laboratory to a hospital, including transporting along highly populated residential corridors and/or along heavily trafficked roadways, should be anticipated in advance. Because of the known latency period between exposure and the development of any clinical manifestations of 
infection that might make the patient potentially infectious to others, special isolation would not normally be required during the time frame immediately following exposure or during the disease incubation period. The need for responsible escorts also should be considered.

6. Healthcare providers must be allowed to make informed and reasonable decisions based on clinical judgment; triage plans should therefore be flexible, to allow for varied situations. The management algorithm should be updated regularly and should include a roster of medical experts who are available for immediate consultation.

7. Pathogen-specific algorithms are vital to the triage process. Expert working groups should be convened to develop such algorithms and to update them as needed, based on advancing knowledge of the underlying disease process and/or the advent of new or more effective treatment modalities.

\section{IsOlation AND MedicAl CARE}

Workshop participants agreed that each BSL- $(3+4)$ laboratory should make provision in advance for the medical care of any employee who becomes infected during the course of research, in a setting that minimizes the risk of transmission of infection to others. Although such a treatment setting has been termed a "Biocontainment Patient Care Unit" (BPCU) in a recent publication, ${ }^{10}$ use of the term "biocontainment" appears to imply that patient care will be performed in a unit resembling a BSL- $(3+4)$ laboratory, rather than in a hospital. There was general agreement at the workshop that the term "biocontainment" should be reserved for laboratories and that hospital facilities for the treatment of infected laboratory workers should be referred to as care and isolation units, or something similar.

\section{Medical Care in a Referral Hospital}

The currently operational BSL- $(3+4)$ laboratories represented at the workshop differ in how they prepare to manage laboratory infections. Since its construction in the early 1970s, USAMRIID has maintained its own small medical unit resembling a small BSL-4 laboratory, in which physicians and nurses can perform patient care while wearing positive-pressure suits. By contrast, all other laboratories have developed memoranda of understanding with local hospitals that have the capacity for treating any employees who become infected with BSL- $(3+4)$ pathogens while employing all necessary infection control procedures. This approach is supported by the fact that infections by most BSL-3 and -4 agents are actually less communicable than such diseases as influenza or XDRtuberculosis, which all hospitals must already be prepared to manage.

\section{Recommendations:}

1. Workshop participants generally recognized that there is no medically based rationale for facilities accommodating patients exposed to BSL-3 or -4 pathogens to be constructed to standards approximating a corresponding containment laboratory. Delivering state-of-the-art medical intensive care in such a setting would present considerable challenges, and it is not clear that the use of such facilities would lead to superior patient care or reduce the risk of exposure for caretakers and the community. Such facilities might also fail to meet current standards for hospital accreditation. In summary, there are legitimate grounds for concern that a "BSL-4 approach" to patient care could detract from the overall quality of care delivered and lead to worse, not better, outcomes.

2. Medical care in a referral hospital with standard respiratory isolation capabilities (in accordance with CDC guidelines for respiratory precautions) meets acceptable minimum standards, if specific accommodations and specialized standard operating procedures are in place to handle such patients. A significant benefit of using a referral hospital isolation suite is the ability to render appropriate medical and diagnostic care in an established hospital setting.

3. Each BSL-3 and BSL-4 laboratory should have a contract or a formal memorandum of agreement with a referral hospital for managing employees with an occupational exposure to a BSL-3 or -4 pathogen.

\section{Minimum Standards for Isolation Units}

Workshop participants agreed that any unit designated for the isolation and treatment of laboratory employees accidentally infected with a BSL-3 or -4 pathogen should be designed to maximize the efficacy of patient care, while minimizing the risk of transmission of infection.

\section{Recommendations:}

1. A risk assessment should be conducted to determine the location, redundancy, and reliability of ventilation systems, waste handling and decontamination procedures, access control plans, and physical security measures. Because risk assessment is driven by locality and 
individual circumstances, there will of necessity be some institution-specific standards. However, crossinstitutional standard operating procedures are essential to convert a hospital isolation room or dedicated ward capable of treating patients with active TB or other respiratory pathogens to one that can safely receive patients who have been exposed to BSL-3 or -4 agents.

2. Although risk assessments will differ by institution, a standardized process should be developed. Performance and procedures should be evaluated on a periodic basis to ensure that the risk of infection to staff and surrounding patient care units continues to be minimized. Periodic drills simulating the handling of a potential exposure case should be performed to train, educate, and update staff as to how such risks can be mitigated.

3. Staff should be sensitive to the emotional impact on patients of being placed in isolation for a potentially prolonged period of observation following a serious laboratory exposure. Psychological support and counseling should be offered.

4. Specific engineering areas to be addressed include:

- Location. Access to the treatment unit must be secure and controlled; it should be separated from public areas, so as to minimize traffic.

- Ventilation. Directional airflow must be employed and monitored by alarm devices. HEPA filtration should be provided for the exhaust air, with regularly scheduled certification as provided by the hospital standard operating procedure. Consideration should be given to requiring HEPA filtration of the supply air, so as to prevent back-flow of contaminated air from the care and isolation unit in the event of system failure. Plans should include performance measures that minimize or prevent re-entrainment into the air supply. Redundancy and complete reliability of the ventilation system should be achieved.

- Power. The facility should be capable of ensuring continuous operation through the rapid provision of emergency power in the event of a power outage.

- Waste. A separate sanitary waste line is not required. Plans should include procedures for handling contaminated waste, including linens, equipment, and patient waste.

- Space/storage. The overall plan for the care and isolation unit should address optimum floor design and the placement and organization of equipment. Adequate space should be allotted for staging, sink areas, waste tracking and storage, eyewash, and storage of gowns, gloves, and other personal protective equipment.

- Decontamination. Appropriate methods of decontamination, including gaseous decontamination of equipment, must be developed and detailed in standard operating procedures. This should include methods to prevent cross-contamination such as vent lines, bathrooms, and vacuum lines. Single-use equipment is preferable, and the re-use should be minimized. A dedicated autoclave is not required, but the unit should have access to an autoclave within reasonable proximity.

5. A plan for providing clinical laboratory services based on available resources should be developed in advance. Laboratories should be identified that have the reagents, capacity (including appropriate levels of biocontainment), and willingness to perform necessary tests. Pointof-care diagnostics should be used whenever possible.

6. Procedures for postmortem examinations and the subsequent handling and transporting of cadavers should be developed. Plans should include consideration of local customs and applicable state laws.

\section{Staff Recruitment and Training}

Responses to the questionnaire and discussions at the workshop indicated a general recognition that the success of a care and isolation unit for the treatment of infections by BSL-3 and -4 agents will be critically dependent on the existence of a dedicated and properly trained staff.

\section{Recommendations:}

1. Because of the understandable concern that medical staff will have about their risk of exposure to a BSL-3 or -4 pathogen, training should be "end-to-end" and include first responders, ambulance drivers, clinical laboratory staff, mortuary workers, and security personnel in addition to the unit staff. Consideration should be given to offering CME credits or special certification as an incentive.

2. Training materials should be developed, including a standardized curriculum, the establishment of a minimum number of training hours, and the conduct of annual drills. Ideally, sessions should be conducted by healthcare providers who are familiar with managing patients with exposures to BSL-3 and -4 agents.

3. Mannequins are important training tools for hands-on experience and regular practice drills. Technological advances such as simulation training should also be used where possible. Outfitting of a mock care and isolation unit that would be available for training specialized teams might also be considered.

\section{Community Outreach}

All workshop participants recognized that the local community should be the focus of educational outreach programs about the scope, design, and operating principles of the care and isolation unit long before any accidental 
exposure to a BSL-3 or -4 agent might occur. It is essential to provide the community with a realistic understanding of the limited transmissibility and the likelihood of successful treatment of most infections by BSL- 3 or -4 pathogens, so as to minimize the likelihood of a panic response.

\section{Recommendations:}

1. Community leaders and media representatives should be invited to tour the unit, interact with staff, observe drills, and see the specialized equipment used to contain infection.

2. In the event of an exposure, all relevant information must be promptly reported in a transparent manner that protects patient confidentiality. It is important to address community concerns, while still prescribing realistic treatment.

3. Patients who do not require full-scale isolation should not be isolated at a higher level than is medically reasonable in response to community fears of disease transmission that lack any scientific basis. Experience has shown that community liaisons who can explain technical issues in an understandable manner are helpful.

\section{Future Initiatives}

Workshop participants agreed that currently active BSL$(3+4)$ laboratories should work together with representatives of new labs that will open over the next few years to develop standard approaches to the detection and management of accidental exposures to virulent pathogens. A number of important areas for collaborative efforts were identified.

\section{Recommendations:}

1. The NIBC should take the lead in inviting representatives from a broad range of BSL- $(3+4)$ laboratories to form a working group to develop consensus statements and algorithms for the management of laboratory exposures to BSL-3 and -4 pathogens and for the evaluation and triage of employees with unexplained illnesses.

2. A working group should be designated to address the urgent need to make IND vaccines available to researchers and support staff of BSL- $(3+4)$ laboratories and to recommend their appropriate use.

3. On a local level, a working group should be designated to consider issues involved in the transport of personnel exposed to BSL-3 and -4 pathogens from the site of exposure in the laboratory to the care and isolation unit in the referral hospital.

4. A working group should be designated to consider how point-of-care laboratory tests in a unit can be made compatible with regulations for quality control in approved hospital laboratories.

The most effective means of dealing with the problem of accidental laboratory infections is to prevent their occurrence through a combination of engineering controls, training in proper lab techniques, employee vaccination, and an active biosafety program. Should an exposure occur despite these efforts, each facility must have a program in place to deal promptly with any eventuality, so as to protect the exposed individual and the rest of the community. Fortunately, biosafety programs at currently operational BSL- $(3+4)$ laboratories have been highly successful in preventing accidental infections and have provided an extensive knowledge base that can be drawn on by the future expanded biodefense community.

\section{ACKNOWLEDgments}

The authors wish to thank the following participants who chaired the breakout sessions and prepared the session reports that became the basis for this consolidated meeting report: Marshall Bloom, Heinz Feldmann, Nancy Hoe, Mark Kortepeter, Frank Kutlak, Jean Patterson, George Risi, and Eli Warnock.

\section{REFERENCES}

1. Homeland Security Presidential Directive (HSPD) 10: Biodefense for the $21^{\text {st }}$ Century. Washington, DC: The White House; April 28, 2004. http://www.homeland.ca.gov/ pdf/ HSPD-10.pdf. Accessed May 11, 2009.

2. Homeland Security Presidential Directive (HSPD) 18: Medical Countermeasures against Weapons of Mass Destruction. Washington, DC: The White House; January 31, 2007. http://www.fas.org/irp/offdocs/nspd/hspd-18.html. Accessed May 11, 2009.

3. Government Accountability Office. High-Containment Biosafety Laboratories. Preliminary Observations on the Oversight of the Proliferation of BSL-3 and BSL-4 Laboratories in the United States. GAO-08-108T. Washington, DC: GAO; Oct 4, 2007. http://www.gao.gov/new.items/d08108t.pdf. Accessed May 11, 2009.

4. Kortepeter MG, Martin JW, Rusnak JM, et al. Managing potential laboratory exposure to ebola virus by using a patient biocontainment care unit. Emerg Infect Dis 2008;14(6): 881-887.

5. Bannister B, Puro V, Fusco FM, Heptonstall J, Ippolito G; EUNID Working Group. Framework for the design and operation of high-level isolation units: consensus of the European Network of Infectious Diseases. Lancet Infect Dis 2009;9(1):45-56.

6. Centers for Disease Control and Prevention, Office of Biosafety. Classification of Etiologic Agents on the Basis of Hazard, 
4th ed. Atlanta: U.S. Department of Health, Education and Welfare, Public Health Service; 1974.

7. Centers for Disease Control and Prevention. Biosafety in Microbiological and Biomedical Laboratories [BMBL], $5^{\text {th }}$ ed. Washington, DC: U.S. Department of Health and Human Services, Public Health Service; 2008. http://www.cdc.gov/ OD/ohs/biosfty/bmbl5/BMBL_5th_Edition.pdf. Accessed May 11, 2009.

8. Le Duc JW, Anderson K, Bloom ME, et al. Framework for leadership and training of biosafety level 4 laboratory workers. Emerg Infect Dis 2008;14(11):1685-1688.

9. Lemon SM, Thaul S, Fisseha S, O’Maonaigh HC, eds. Committee on a Strategy for Minimizing the Impact of Naturally Occurring Infectious Diseases of Military Importance: Vaccine Issues in the U.S. Military [authors]. Protecting Our Forces: Improving Vaccine Acquisition and Availability in the U.S. Military. Washington, DC: National Academies Press; 2002: ch. 3.
10. Smith PW, Anderson AO, Christopher GW, et al. Designing a biocontainment unit to care for patients with serious communicable diseases: a consensus statement. Biosecur Bioterror 2006;4(4):351-365.

Manuscript received January 6, 2009;

accepted for publication March 5, 2009.

Address correspondence to: Peter Jahrling, PhD Director

NIAID Integrated Research Facility 8200 Research Plaza Frederick, MD 21702

E-mail: jahrlingp@niaid.nih.gov 